\title{
Un enfoque basado en juegos educativos para aprender geometría en educación primaria: Estudio preliminar
}

\author{
Antonio-Joaquín Franco-Mariscal ${ }^{1}$ \\ ORCID: 0000-0002-8704-6065 \\ Paola Simeoli Sánchez ${ }^{1}$ \\ ORCID: 0000-0002-3669-1742
}

\section{Resumen}

El aprendizaje de la geometría es primordial en matemáticas para identificar las figuras geométricas y aprender sus propiedades. El empleo de juegos educativos en el aula, ofrece nuevas oportunidades para motivar a los estudiantes y aprender matemáticas en la vida diaria desde una perspectiva socio-constructivista dentro de las posturas falibilistas y cuasi-empiristas. El objetivo de este trabajo es diseñar y evaluar una secuencia didáctica sobre geometría para primaria, donde la base del aprendizaje se articule a través de diferentes juegos educativos integrados de manera conjunta en la secuencia, permitiendo comparar el aprendizaje adquirido con los resultados de tareas más tradicionales. El estudio preliminar se desarrolló con 13 estudiantes de 7 años de un colegio de Málaga (España). Para cada contenido, se incluyeron en la secuencia tareas tradicionales (con fichas de trabajo con escasas oportunidades en el contexto social) y juegos (games y task involving play - TIP o tareas que promueven escenarios entre play y game-). El análisis cualitativo y cuantitativo de cada tarea mediante categorías y el test de Wilcoxon mostraron que los juegos educativos resultan más favorables para el aprendizaje de la geometría que los contenidos planteados con una tarea tradicional, en particular, aquellas relacionadas con la identificación de polígonos. Los resultados apuntan a que el tipo de juego educativo que resulta más efectivo en el aprendizaje de la geometría son los TIPs, ya que sólo en éstos se obtuvieron diferencias estadísticamente significativas al compararlos con tareas tradicionales.

\section{Palabras clave}

Geometría - Educación primaria - Juegos Educativos - Tareas que Implican Juegos (TIP) - Tareas tradicionales.

1- Universidad de Málaga, Málaga, España.Contactos: anjoa@uma.es; pao_ss@hotmail.es 


\section{An educational game-based approach to learning Geometry in Elementary School: A preliminary study}

\section{Abstract}

Learning geometry is paramount in mathematics to identify geometric shapes and learn their properties. The use of educational games in the classroom offers new opportunities to motivate students and to learn mathematics in daily-life contexts from a socioconstructivist perspective within the fallibilist and quasi-empiricist positions. The aim of this paper is to design and evaluate a teaching unit on geometry for elementary school, where the basis of learning is articulated through different educational games integrated together in the sequence, allowing us to compare the acquired learning with the results of more traditional tasks. The preliminary study was developed with 13 seven-year-old students from a school in Malaga (Spain). For each content, traditional tasks (worksheets with scarce opportunities in the social context) and games (together with task involving play - TIP or tasks promoting scenarios between play and game) were included in the teaching unit. The qualitative and quantitative analysis of each task using categories and the Wilcoxon test showed that educational games are more favorable to the learning of geometry than the contents proposed with a traditional task, in particular, those related to the identification of polygons. The results suggest that the type of educational game that is most effective in learning geometry is TIP, since only in these games statistically significant differences were obtained when compared to traditional tasks.

\section{Keywords}

Geometry - Elementary school - Educational games - Task Involving Play (TIP) Traditional tasks.

\section{Introducción}

De todas las ramas de la Matemática, la Geometría es una de las más intuitivas, concretas y vinculadas a la realidad (International Commission on Mathematical Instruction, 1998, p. 337). Es precisamente, esta conexión con la vida diaria la que no debe perderse al enseñar matemáticas desde edades tempranas, ya que estaríamos contribuyendo a producir una desmotivación por esta materia cuando el propio alumno/a no ve ninguna relación con el mundo en el que vive. Éste es, sin duda, uno de los mayores retos a los que se enfrenta un profesor de matemáticas, ya que algunos estudios indican que el uso de una metodología tradicional que utiliza en el aula una imagen alejada de la visión humanística de las matemáticas escolares y más cercana a la filosofía absolutista, produce en los estudiantes un cierto rechazo hacia la disciplina debido a su aparente complejidad, carácter abstracto y poco motivador (MINERVA TORRES, 2001). 
Diferentes autores e instituciones (OHIO..., 2004; DE GUZMÁN, 2004; MUÑIZ ALONSO; RODRÍGUEZ, 2014) coinciden en que se pueden conseguir mejores resultados en el aprendizaje de los estudiantes, si se emplean en el aula metodologías innovadoras durante el proceso de enseñanza desde las primeras etapas de la educación, siendo también fundamental la interacción entre alumnos, estudiantes y profesor. Ohio Department of Education (2004) afirma que los logros de los estudiantes sobre geometría y competencia espacial pueden ser alcanzados en ambientes adecuados con estrategias paralelas al desarrollo de las características de los niños, donde el profesor presenta oportunidades para que aprendan por ellos mismos (por ejemplo, creando diferentes figuras geométricas en actividades artísticas, diseñando figuras con bloques o clasificando bloques similares). La visión de Piaget refuerza este sentido enfatizando que la geometría debe ser enseñada a través de actividades manuales. Según Clements (1999) el estudiante debe ser "absorbido" en actividades de geometría no como un deber o bajo presión, sino como diversión. En este sentido, el uso de juegos educativos en el aula se ha mostrado como una herramienta eficaz para producir estos logros, esto no significa que sea el único enfoque para este objetivo, existen otros como: resolución de problemas, investigaciones matemáticas, etc. que resultan también muy productivos.

El objetivo de este trabajo es diseñar y evaluar una secuencia didáctica sobre geometría para primaria, donde la base del aprendizaje se articule a través de diferentes juegos educativos integrados de manera conjunta en la secuencia, permitiendo comparar el aprendizaje adquirido con los resultados de tareas más tradicionales. Este se considera un aspecto novedoso de esta propuesta, ya que es difícil encontrar en la literatura secuencias didácticas completas, evaluadas y organizadas en torno a juegos educativos, ya que habitualmente este tipo de tareas se diseñan de forma aislada.

\section{Marco teórico}

\section{El papel de los juegos en la enseñanza y aprendizaje de las matemáticas}

El uso de juegos como herramienta para fomentar el aprendizaje, se ha explorado ampliamente en el campo de la educación en general y de la educación matemática en particular. Este enfoque suele usarse con frecuencia en las etapas infantil y primaria, siendo su uso poco extendido en la educación secundaria y universitaria.

La revisión de la literatura muestra que las investigaciones sobre juegos hacen una distinción entre play y game. Sin embargo, establecer una diferencia clara entre ambos términos no es sencillo, ya que dependen de numerosos factores, a veces transitorios y contradictorios, así como de las cualidades del contexto al que se apliquen, en ese sentido se utilizarán estos dos términos para referirnos al juego.

Play se define habitualmente en términos de su opuesto, es decir, de un trabajo serio (GOODALE; GODBEY, 1988). Los límites aceptados comúnmente entre ambos a menudo suelen ser difusos o se rompen durante el play, lo que hace difícil diferenciar entre trabajo y play (HUIZINGA, 2000). Sin embargo, el proceso de aprendizaje requiere de ambas condiciones (Bakhtin, 1984), y éstas incluyen el conocimiento formal e informal con actividades que implican a menudo play. Chazan (2002) sugirió que play ocupa un ámbito que queda fuera 
de los eventos cotidianos, tiene que ver con la imaginación y el razonamiento, donde todo es posible. Estas definiciones apuntan a que play puede considerarse en diferentes vertientes. A pesar de la variedad de definiciones que se pueden encontrar sobre play, Henricks (1999) definió sus principales características: a) play es una experiencia, b) produce una motivación intrínseca más que extrínseca, c) el proceso es más importante que el resultado final, y d) implica algún tipo de nivel de compromiso activo.

La distinción entre play y game generalmente se vincula a los conceptos de paidia y ludus propuestos por Caillois (2001) como dos polos opuestos de las actividades lúdicas. Paidia (o play) denota una más libre, expresiva, improvisada, incluso tumultuosa recombinación de comportamientos y significados, mientras que ludus (o game) se asocia con un juego estructurado con reglas y conflictos competitivos con la finalidad de llegar a un objetivo. Dempsey et al. (2002) definieron game como un conjunto de actividades, que implican uno o más jugadores, que tiene unos objetivos, unas restricciones, una recompensa y unas consecuencias, es guiado por reglas e implica algunos aspectos de competición consigo mismo. En la definición de Salen y Zimmerman (2004), game es un sistema en el que los jugadores se involucran en conflictos artificiales, definidos por reglas, que derivan en resultados cuantificables. Por tanto, cualquier game plantea un componente de desafío en términos de reglas y propósitos, que puede corresponder con un desafío personal o competitivo (CHAMOSO et al., 2004).

Como se aprecia, las definiciones entre play y game son amplias. Sin embargo, las cualidades que las describen están estrechamente relacionadas con algunos aspectos de buenas prácticas en la enseñanza de las matemáticas. Así, el juego se percibe como beneficioso para el desarrollo y la promoción de la creatividad, la imaginación y el aprendizaje espontáneo (BRUNER, 1986; ALSINA, 2007), ofreciendo nuevas oportunidades para la enseñanza. Para Minerva Torres (2002) el uso de games también puede fomentar y estimular ciertas cualidades morales en los alumnos como el autocontrol, la honestidad, la seguridad, la atención y la concentración en el desarrollo de las tareas, la reflexión, la búsqueda de alternativas para ganar, el respeto a las reglas, la iniciativa, el sentido común, el sentido de la solidaridad con los compañeros, y, sobre todo, el juego limpio. Esta autora también cree que la práctica de games en el aula introduce la competición como estímulo para el aprendizaje. Por otro lado, las implicaciones de tipo emocional, el carácter lúdico, la desinhibición, son fuentes de motivación que proporcionan un acercamiento al aprendizaje de una forma distinta a la tradicional (CORBALÁN; DEULOFEU, 1996).

Otros investigadores argumentan que la motivación fundamental para cualquier game debe ser aprender, sirviendo éste como un modo eficaz para hacerlo (BELANICH; ORVIS; SIBLEY, 2004). En este sentido, los games pueden ser sustitutos muy efectivos de las tareas más tradicionales en todos los niveles educativos (MONDEJA et al., 2001; EBNER; HOLZINGER, 2007). Asimismo, el enfoque didáctico basado en games tiene cabida dentro del marco constructivista del aprendizaje (DRIVER; OLDHAM, 1986; ERNEST, 1994), ya que uno de los requisitos del constructivismo es que los estudiantes se involucren en aprender conocimientos considerados difíciles. Tales desafíos permiten que cada estudiante pueda construir su propio conocimiento, lo que se podría favorecer con métodos de enseñanza alternativos como el uso de games en el aula. 
Concretamente, este trabajo se enmarca en la perspectiva del socio-constructivismo de Ernest (1991, 1996, 2004) incluido dentro de las posturas falibilistas y cuasi-empiristas (HANDAL, 2003), y está asociado con el pensamiento posmodernista de la educación, la filosofía y las ciencias sociales. Se enfatiza, por tanto, la práctica humana de las matemáticas, opuesta a la imagen absolutista. Según este enfoque el estudiante construye teorías a partir de su experiencia y luego éstas se ajustan cuando se someten a otras experiencias reales. Para el constructivismo el énfasis está en el sujeto epistémico, en otras palabras, el profesor no transmite conocimiento hace que el estudiante "les enseñe cómo desarrollar su cognición" (CONFREY, 1992). Es decir, se le presentan al estudiante oportunidades para modificar estructuras de forma que lleguen a ser compatibles con las expectativas y fines del instructor. Desde este punto de vista, la autonomía del alumno es crucial, contraria a una visión tradicional que concibe al estudiante de manera pasiva. De este modo, los juegos pueden contribuir a ofrecer a los estudiantes una imagen de las matemáticas escolares humanizadas y anti-absolutistas.

Por otra parte, un aspecto fundamental de la educación es la construcción del pensamiento crítico (OSBORNE, 2014; BLANCO; ESPAÑA; GONZÁLEZ; FRANCOMARISCAL, 2015). Respecto a este, los alumnos pueden llegar a desarrollarlo realizando actividades basadas en el razonamiento y en la reflexión, que se pueden potenciar con games. Los games se pueden emplear también para fomentar el interés de los alumnos y asegurar su participación en el proceso de aprendizaje. De esta forma, los trabajos de Orlik (2002) sobre metodologías activas en la enseñanza de las ciencias en general, mostraron los games como uno de los enfoques más importantes.

Desde el punto de vista educativo, si se desea emplear games en actividades de aula, la función didáctica debe superponerse al placer del entorno lúdico. Así, los games pueden constituir un puente entre la enseñanza formal de las matemáticas y la enseñanza de esta en ámbitos informales (BLANCO, 2004). Mondeja et al. (2001) definieron un conjunto de cualidades que deben tener los games para que sean útiles en la enseñanza y aprendizaje: (a) deben impulsar la actividad de los alumnos en una variedad de caminos dentro de la organización de la enseñanza y una vez motivados los estudiantes deben desarrollar su actividad cognitiva, consolidando su aprendizaje de una forma activa; (b) deben mejorar indirectamente la eficiencia del proceso educativo al requerir una actividad más reflexiva $\mathrm{y}$ (c) deben llevarse a la práctica de una forma bien planificada en consonancia con los objetivos educativos y sus implicaciones en el aula.

Un estudio reciente (FRANCO-MARISCAL et al., 2016, p. 1178), ha identificado un nuevo tipo de recurso relacionado con los juegos, denominado TIP (Task Involving Play), que parece producir buenos resultados de aprendizaje en el ámbito de las ciencias, a la vez que permite evaluar de una forma más precisa los juegos educativos no digitales desde el punto de vista de su naturaleza. En este sentido, un juego podría clasificarse como play o game, pero también como un término medio, TIP. De acuerdo con estos autores, los recursos TIPs plantean al alumno escenarios intermedios entre play y game, que deben fomentar la creatividad artística o tecnológica del estudiante con un papel activo, estar directamente relacionado con su vida cotidiana y fomentar el trabajo en equipo. Otras características de los TIPs que contribuyen al aprendizaje son juegos simples y atractivos que los alumnos consideren útiles para aprender. Algunos ejemplos, reconocidos son la realización de un dibujo o la construcción de una maqueta para resolver un problema cotidiano. Sin duda, 
esta conexión de las matemáticas con la vida diaria está íntimamente relacionada con el enfoque competencial que plantean los currículos actuales (ESPAÑA..., 2014) y los programas de evaluación como PISA (OECD, 2016), los cuales pretenden que el alumnado desarrolle la capacidad para aplicar el conocimiento matemático a problemas y situaciones de su vida diaria. Diversos autores coinciden en que los juegos educativos pueden constituir una buena estrategia para que los estudiantes desarrollen competencias de una materia atractiva (FRANCO MARISCAL; VIDAL, 2013; MUÑIZ; ALONSO; RODRÍGUEZ, 2014).

Por otro lado, el uso de juegos educativos en el aula contribuye asimismo a la iniciativa planteada por las Naciones Unidas (1996), que reconoció las actividades recreativas como una de las diez esferas prioritarias en el desarrollo infantil y juvenil como un elemento necesario para prevenir y combatir problemas sociales como las drogas o la delincuencia (VARELA; GRADAÍLLE; TEIJEIRO, 2016).

Finalmente, debemos indicar que los juegos no han escapado al avance de las nuevas tecnologías, lo que ha producido en los últimos años una rápida proliferación de juegos de ordenador y vídeo. Esta tendencia, conocida como gamificación (DETERDING et al., 2011) ha trascendido del entretenimiento al mundo educativo (WAYER; CRIPPEN; DAWSON, 2015). La gamificación no será objeto de estudio en este trabajo que se centrará solo en juegos no digitales.

\section{Juegos educativos en la enseñanza de las matemáticas}

Dentro de la matemática escolar, la variedad de tipos de games es muy amplia, encontrándose en la literatura diferentes criterios para clasificarlos. Según Farias y Rojas (2010), games se pueden clasificar según la conducta lúdica que manifieste el alumno en: a) de función, b) de ficción, c) de construcción, o d) de agrupamiento o representación del entorno. Otros criterios de clasificación son el propósito y la forma o su estructura (CHAMOSO et al., 2004; MILLAR, 1992, entre otros). Así, games se clasifican en: a) cooperativos, b) libres o espontáneos, c) de reglas o estructurados, d) de estrategias, e) de simulación, f) de estructuras adaptables, y g) populares y tradicionales. Otra posible clasificación es la de Olfos y Villagrán (2001) que distinguen los games en: a) pre, co y post instrucción, b) de conocimiento y de estrategia, c) con lápiz, papel, calculadoras y fichas (ajedrez), d) de numeración, cálculo, cuentas, operaciones, criptogramas, series, adivinanza de números, con el sistema métrico y la divisibilidad, y e) juegos aritméticos, algebraicos, geométricos, topológicos, manipulativos y lógicos.

El trabajo de Villarroel y Sgreccia (2011) sobre caracterización de materiales didácticos para la enseñanza de la geometría, logró identificar siete grandes grupos: modelos fijos 2D y 3D, rompecabezas geométricos, tangram, geoplano, transformaciones dinámicas, origami o papiroflexia y objetos del entorno real. Como puede observarse, muchos de ellos se asocian a juegos educativos y según estos autores son de utilidad en la enseñanza de la geometría.

Independientemente de la clasificación a la que se inscriba el juego, algunos autores coinciden en que el juego contribuye a un mejor aprendizaje (CORBALÁN, 1994; DE GUZMÁN, 2004; ROJAS, 2009; CANO; ZAPATA, 2010), lo que ha producido que en la literatura hayan emergido un gran número de juegos o propuestas educativas para la 
enseñanza y aprendizaje de las matemáticas (BUENO; MONTEOLIVA, 1993; ALSINA, FORTUNY; PÉREZ, 1997; FARIAS; ROJAS, 2010). La mayoría de estos juegos educativos se limitan simplemente a la presentación de una propuesta y en algunos casos a una valoración cualitativa del docente acerca de sus impresiones sobre el aprendizaje de los estudiantes. Salvo contadas excepciones, dichas propuestas no incluyen un apartado de evaluación de los materiales innovadores que se proponen, en el que se investigue el efecto que produce su implementación y las causas responsables de la mejora en el aprendizaje que algunos autores indican (DURÁN et al., 2004; FARIAS; ROJAS, 2010; MUÑIZ; ALONSO; RODRÍGUEZ, 2014). Aún más, no se han encontrado propuestas completas en las que se utilicen y evalúen un conjunto amplio de juegos de naturaleza game y TIPs en una misma secuencia, como la que aquí se presenta.

Es por ello, que este trabajo le apuesta a la incorporación de juegos educativos en el proceso de enseñanza y aprendizaje de las matemáticas como una potente herramienta que puede dar frutos en el ámbito educativo, investigando su efecto en un estudio preliminar con estudiantes españoles del segundo curso de primaria en el campo de la geometría.

\section{Diseño de la investigación}

\section{Objetivo de la investigación}

El problema que se plantea en este trabajo consiste en investigar, en qué medida un enfoque didáctico basado en juegos educativos integrados dentro de una secuencia didáctica puede contribuir al aprendizaje de alumnos de 7 años ( $2^{\circ}$ curso de educación primaria en España) en el ámbito de la geometría en comparación con una metodología tradicional. Concretamente, se plantean dos preguntas de investigación:

- Pregunta de investigación 1: ¿Existen diferencias en los resultados de aprendizaje de los estudiantes cuando aprenden un concepto geométrico a través de juegos educativos frente a una metodología tradicional?

- Pregunta de investigación 2: ¿Qué tipos de juegos educativos (games o TIPs) producen mejores resultados en el aprendizaje de la geometría?

\section{Participantes}

Se realizó un estudio preliminar con una muestra de 13 estudiantes de edades comprendidas entre 7 y 8 años del $2^{\circ}$ curso de educación primaria de un colegio de Benalmádena (Málaga, España). El 38\% de los estudiantes eran niñas y el 62\% niños. Dichos alumnos desarrollaron la secuencia didáctica con la segunda autora de este artículo durante el curso 2014-15.

\section{Diseño de la secuencia didáctica basada en juegos educativos}

Para responder a las preguntas de investigación se diseñó y llevó a la práctica la secuencia didáctica titulada "Las figuras geométricas planas" en 7 sesiones de 1 hora de duración. Se pretende que el estudiante, al terminar la unidad, sea capaz de alcanzar los 
siguientes objetivos didácticos: 1) conocer el concepto de figura geométrica, 2) reconocer, comparar y diferenciar líneas poligonales rectas y curvas, abiertas y cerradas, 3) reconocer diferentes polígonos en elementos del entorno de la vida diaria, 4) reconocer y diferenciar la circunferencia del círculo, 5) conocer y diferenciar los lados y los vértices como elementos básicos de una figura plana, y 6) valorar que las figuras geométricas juegan un papel importante en la vida diaria, su reconocimiento y uso es fundamental para el desarrollo de la actividad humana.

Los contenidos abordados en el transcurso de la secuencia didáctica se muestran en la Tabla 1.

Tabla 1- Tipos de contenidos de la secuencia didáctica sobre geometría

\begin{tabular}{|c|c|c|}
\hline Conceptual & Procedimental & Actitudinal \\
\hline Figuras geométricas. & $\begin{array}{l}\text { Reconocer diferentes figuras geométricas en una } \\
\text { fotografía. }\end{array}$ & $\begin{array}{c}\text { Valorar los usos de las figuras geométricas en } \\
\text { la vida diaria. }\end{array}$ \\
\hline $\begin{array}{l}\text { Líneas poligonales y curvas, } \\
\text { abiertas o cerradas. }\end{array}$ & $\begin{array}{l}\text { Reconocer, comparar y diferenciar entre sí líneas } \\
\text { poligonales y curvas, abiertas o cerradas. }\end{array}$ & $\begin{array}{l}\text { Valorar el uso de los tipos de líneas en el } \\
\text { entorno del alumno. }\end{array}$ \\
\hline Circunferencia y círculo. & $\begin{array}{l}\text { Clasificar objetos de su entorno con forma de } \\
\text { circunferencia o círculo. }\end{array}$ & $\begin{array}{l}\text { Interés por la identificación de circunferencias y } \\
\text { círculos en objetos cotidianos. }\end{array}$ \\
\hline Identificación de polígonos. & $\begin{array}{l}\text { Construir polígonos con palillos. } \\
\text { Construir un polígono con el cuerpo de varios alumnos. } \\
\text { Manipular las piezas del tangram para construir una } \\
\text { figura dada, y reconocer los polígonos utilizados. }\end{array}$ & $\begin{array}{l}\text { Sensibilidad y gusto por la representación de } \\
\text { construcciones geométricas sencillas. }\end{array}$ \\
\hline $\begin{array}{l}\text { Elementos de un polígono: } \\
\text { Lados y vértices. }\end{array}$ & $\begin{array}{l}\text { Diferenciar y contar los lados y los vértices de una } \\
\text { figura plana. }\end{array}$ & $\begin{array}{l}\text { Interés por la identificación de los elementos de } \\
\text { un polígono a partir de los objetos del entorno. }\end{array}$ \\
\hline
\end{tabular}

Fuente: Creación propia.

El diseño de esta secuencia se organizó en torno a un conjunto amplio de juegos educativos. Para cada contenido abordado la secuencia incluye, al menos, una tarea tradicional y otra basada en juegos, lo que permite comparar resultados de aprendizaje entre ambas propuestas.

Como se ha indicado, se entiende como metodología tradicional aquella que emplea una imagen alejada de la visión humanística de las matemáticas escolares. Las tareas tradicionales se desarrollan con el uso de fichas de trabajo, donde se ofrecen escasas oportunidades creadas por el contexto social, no se potencia la creatividad del estudiante y no se conectan las matemáticas con la realidad del alumno. En las clases con juegos educativos se emplearon dos tipos (games y TIPs). En esta investigación game es entendido como una tarea lúdica gobernada por una serie de reglas y propósitos, con un carácter competitivo con la consecución de unos objetivos de acuerdo con las definiciones de Caillous (2001); Mondeja et al. (2001); Dempsey et al. (2002); Salen; Zimmerman, (2004). Concretamente se utilizan dos games: tangram y palabra tabú. Se entiende como TIP la definición dada por Franco-Mariscal et al. (2016) como escenario entre play y game que supone algún tipo de creatividad artística o tecnológica en un contexto de la vida diaria. 
La Tabla 2 presenta los juegos educativos incluidos en la secuencia y el escenario en el que se desenvuelven.

Tabla 2- Juegos educativos de la secuencia didáctica

\begin{tabular}{|c|c|c|c|}
\hline Tarea & $\begin{array}{l}\text { Tipo de juego } \\
\text { educativo }\end{array}$ & Escenario de la vida diaria & Tarea demandada \\
\hline TIP1. Imaginamos el cuento & $\mathrm{TIP}$ & Lectura de un cuento & $\begin{array}{l}\text { Utilizar la imaginación para crear un dibujo } \\
\text { a partir de un cuento }\end{array}$ \\
\hline TIP2. El país de las líneas & TIP & Expresión corporal & Crear líneas con el cuerpo \\
\hline TIP3. La bolsa mágica & TIP & $\begin{array}{l}\text { Sensibilidad hacia las personas con } \\
\text { discapacidad }\end{array}$ & Identificar un objeto por su forma \\
\hline TIP4-1. Jugamos con palillos & $\mathrm{TIP}$ & $\begin{array}{l}\text { Utilizar palillos de dientes como } \\
\text { objetos cotidianos para jugar }\end{array}$ & Crear polígonos con palillos de madera \\
\hline TIP4-2. Nos vamos al patio & TIP & Expresión corporal & Crear figuras geométricas con el cuerpo \\
\hline G5. Jugamos con el tangram & Game & Juego infantil & $\begin{array}{l}\text { Construir una figura reconocible a partir } \\
\text { de varias piezas }\end{array}$ \\
\hline G6. La palabra tabú & Game & Juego infantil & $\begin{array}{l}\text { Describir un polígono sin poder usar } \\
\text { ciertas palabras }\end{array}$ \\
\hline
\end{tabular}

Fuente: Creación propia

\section{Metodología didáctica en el aula}

La metodología que se llevó a cabo en el transcurso de la secuencia se sitúa dentro del marco socio-constructivista (DRIVER; OLDHAM, 1986; ERNEST, 1991, 2004) y fomenta el carácter activo y participativo de los estudiantes con el empleo de un enfoque basado en juegos educativos para lograr un aprendizaje significativo.

Antes de realizar las tareas, la docente dio algunas pinceladas de los contenidos matemáticos a abordar, de forma que es el propio alumnado quién debe extraer las conclusiones en la puesta en común que se hace tras las tareas. Como el número de estudiantes es reducido, favorece que todos puedan argumentar su opinión. En cada sesión se realizó en primer lugar la tarea basada en juegos debido a que requería más tiempo, y posteriormente la tradicional al ser más mecánica. Asimismo, se emplearon distintos tipos de agrupamientos en los estudiantes (individual, por parejas, pequeño grupo y gran grupo).

\section{Tareas}

Este apartado describe las 13 tareas desarrolladas (Tabla 3). Como se observa, se propone una actividad tradicional y otra basada en juegos para cada contenido. Para su posterior análisis, a cada una de las actividades se le asignó un código con una letra (T, G o TIP), que indica su carácter tradicional, game o tarea que implica play, respectivamente, seguido de un número. 
Tabla 3- Actividades tradicionales y lúdicas incluidas en la secuencia didáctica

\begin{tabular}{|c|c|}
\hline Tareas tradicionales & Tareas basadas en juegos \\
\hline \multicolumn{2}{|c|}{ Concepto de figura geométrica } \\
\hline $\begin{array}{l}\text { T1. Las figuras } \\
\text { El estudiante debe identificar y marcar diferentes figuras } \\
\text { geométricas (triángulo, cuadrado, rectángulo, pentágono y } \\
\text { círculo) en una fotografía del exterior de una casa con su } \\
\text { jardín. La fotografía ofrece la posibilidad de encontrar todas } \\
\text { las figuras en múltiples lugares. }\end{array}$ & $\begin{array}{l}\text { TIP1. Imaginamos el cuento } \\
\text { El estudiante lee un cuento sobre las figuras geométricas (Salcedo, 2012). Se } \\
\text { pide al alumno que ponga un título a la historia y realice un dibujo en relación } \\
\text { al contenido. El estudiante puede dibujar las figuras geométricas que han } \\
\text { aparecido en el cuento o combinarlas formando una casa, un sol, etc. }\end{array}$ \\
\hline
\end{tabular}

Diferencia entre líneas poligonales 0 curvas, abiertas 0 cerradas

\section{T2. Encontramos las líneas}

Realización de una ficha, en la que el alumno debe identificar y colorear los distintos tipos de líneas (poligonal abierta 0 cerrada, o línea curva abierta o cerrada) con el color asignado Pinta del color que corresponda.

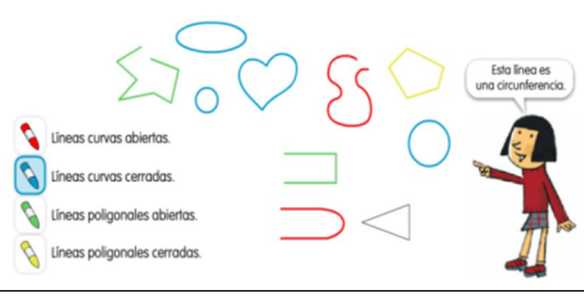

\section{TIP2. El país de las líneas}

Se le da al alumno un conjunto de tarjetas en las que aparecen los diferentes tipos de líneas con un dibujo y su nombre. Se pide a los estudiantes que la representen en el aula y al resto que la identifique.

Diferencia entre circunferencia y círculo

\section{T3. Circunferencia y círculo}

El estudiante debe representar en un dibujo dos objetos que sean una circunferencia y otros dos que sean un círculo, mostrando sus diferencias.

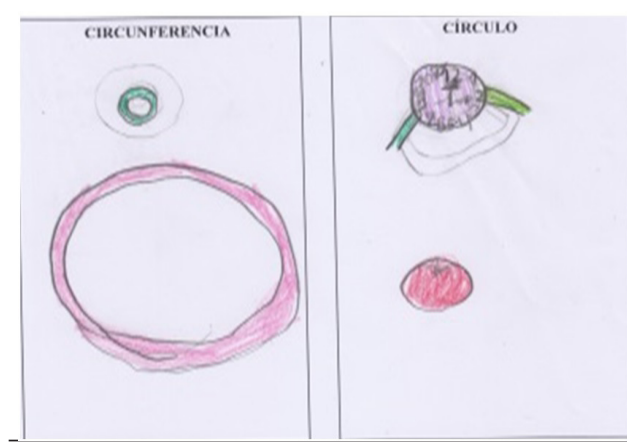

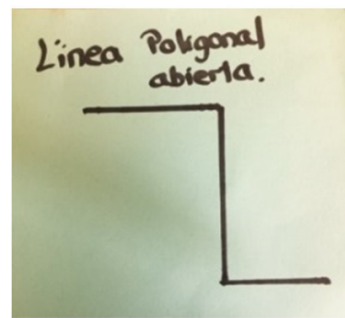

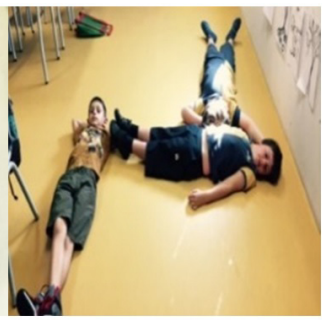

TIP3. La bolsa mágica

Se utiliza una bolsa mágica que contiene diferentes objetos con la forma de una circunferencia (anillo, cinta adhesiva, etc.) o un círculo (tapón de un bote, botón, etc.). Se pide que el alumno con los ojos cerrados como si se tratara de una persona ciega toque un objeto y reconozca si su superficie corresponde a una circunferencia o un círculo.

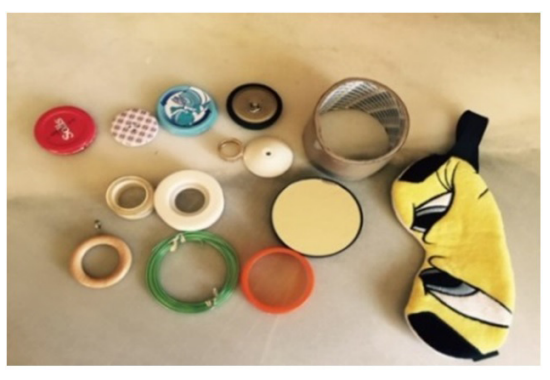


T4. Los polígonos

Realización de una ficha, en la que se debe indicar el nombre de distintos polígonos dada la figura geométrica.

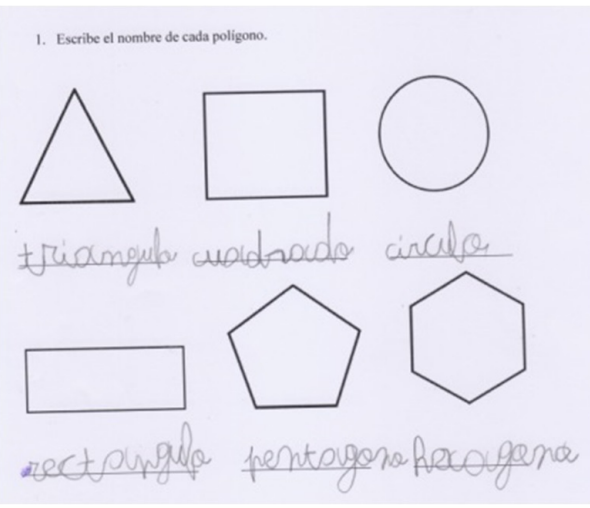

T5. División de figuras

Realización de una ficha en la que se proporcionan al estudiante 7 figuras para que divida 4 de ellas en varios polígonos. Por ejemplo, podría dividir el triángulo en dos triángulos, el rectángulo en cuatro triángulos o en dos rectángulos, etc.

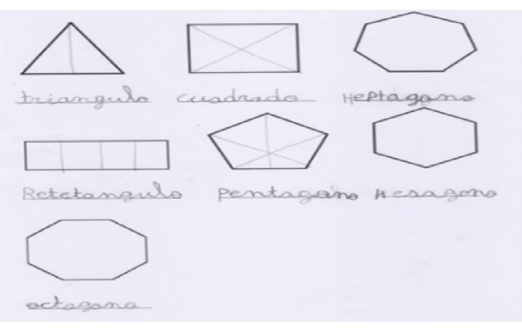

Se reparte a cada estudiante un folio y un sobre que contiene 24 palillos de colores. El estudiante debe formar los siguientes polígonos: triángulo, cuadrado, rectángulo, pentágono y hexágono utilizando todos los palillos dados y pegarlos el folio.

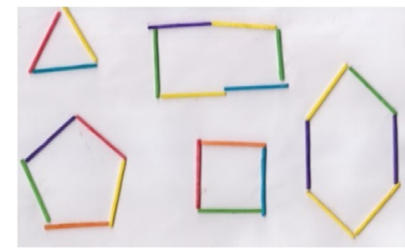

TIP4-2. ¡Nos vamos al patio!

Esta actividad se realiza en el patio y se divide la clase en dos grandes grupos. La profesora nombra una figura geométrica y pide a los estudiantes de cada grupo que la represente. Gana el grupo que consiga formarla en menos tiempo.

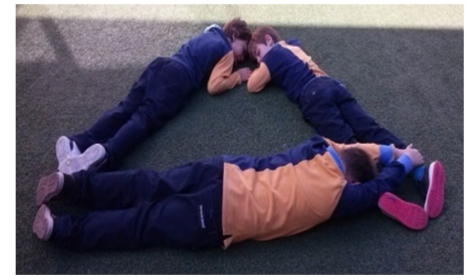

G5. Jugamos con el tangram

Se reparte a cada estudiante un tangram y una ficha con la figura a realizar Se pide representar la figura con las piezas del tangram sin sobreponerlas y anotar el nombre de las piezas utilizadas.

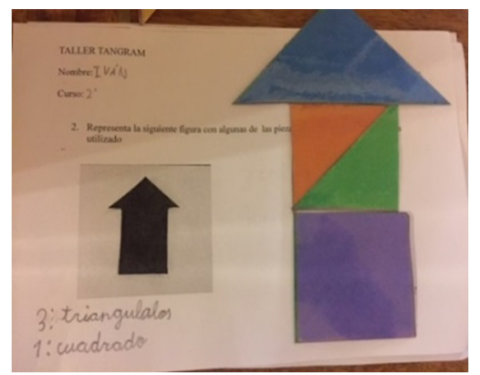

\section{Identificación de los elementos de un polígono}

T6. Los elementos de un polígono

Realización de una ficha donde el estudiante debe identificar los lados y vértices de un triángulo, un pentágono, un rectángulo, un hexágono y un octógono.

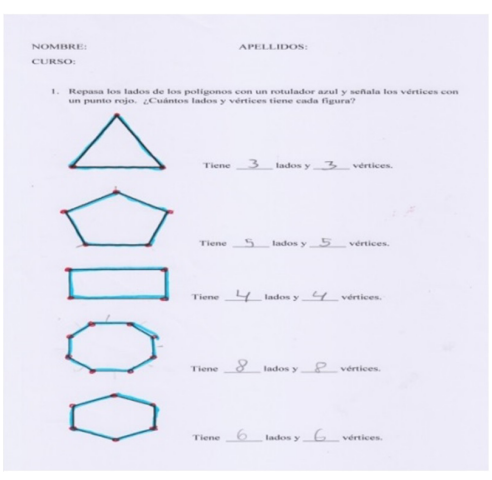

G6. La palabra tabú

Esta actividad se realiza en grupos de cuatro alumnos con una serie de paletas con la forma de un polígono y un conjunto de tarjetas con el dibujo de un polígono y una palabra tabú (ver figura). Todos los grupos disponen de las 6 paletas. Sale un voluntario y coge una tarjeta, la muestra al resto de compañeros y pregunta ¿qué figura es? Los otros grupos deben averiguar de qué figura se trata sin utilizar la palabra tabú. Por ejemplo, si la figura es el triángulo y la palabra tabú "lados", los grupos podrían decir que tiene tres vértices, pero no que tiene tres lados. Para ello, tienen que levantar la paleta de la figura correspondiente. El grupo que lo averigüe primero es el ganador.

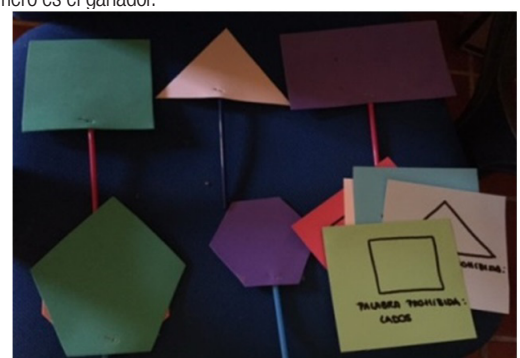




\section{Instrumentos de recolección de datos}

Para evaluar el aprendizaje de los estudiantes se analizaron los resultados de las distintas tareas tradicionales y basadas en juegos. El análisis de datos tuvo dos partes, una primera de carácter cualitativo, que sirvió para definir un sistema de categorías de las respuestas aportadas en cada tarea, estableciéndose cuatro categorías (A, B, C y D) desde la respuesta más adecuada a la más inadecuada en cada uno de los contenidos abordados. El segundo análisis consistió en un tratamiento posterior de tipo cuantitativo basado en el análisis de frecuencias y el establecimiento de comparaciones. El tratamiento de la información se llevó a cabo con el programa estadístico SPSS 21.0 recurriendo a análisis de tipo descriptivo y pruebas de comparación de tipo no paramétrico, como el test de Wilcoxon. Esta es una prueba válida para variables dependientes o repetidas, lo que permite establecer si existen diferencias en el aprendizaje entre el tipo de tareas empleadas.

Se utilizó también el diario del profesor-observador para registrar dos aspectos: a) el progreso de cada sesión y las discusiones que tuvieron lugar en ellas, basadas en notas de campo, y b) las reflexiones sobre el proceso de enseñanza y aprendizaje que estaba teniendo lugar.

\section{Resultados y discusión}

En este apartado se comentan los resultados de cada tarea desarrollada en el aula, presentando algunos ejemplos de las producciones de los estudiantes. La Tabla 4 recoge los datos de todas las tareas y el análisis estadístico.

\section{Concepto de figura geométrica}

En las tareas T1 y TIP1 se pedía al alumno identificar en un dibujo o representar las figuras geométricas más conocidas (triángulo, cuadrado, rectángulo, círculo y pentágono) a partir de una historia. Estas tareas se analizaron atendiendo al número de figuras geométricas que el estudiante fue capaz de identificar, representar y combinar en un dibujo según estas categorías: A) 5 figuras diferentes, B) 5 figuras, pudiéndose repetir algunas de ellas, C) 3 o 4 figuras diferentes, y D) menos de 3 figuras distintas. Debemos indicar que en la tarea TIP1 no aparece en el cuento el pentágono, por lo que en las categorías A y B se valoró el uso de las 4 figuras en más de una ocasión.

Las figuras 1 y 2 presentan ejemplos de las producciones de los alumnos en las distintas categorías para la tarea tradicional (T1) y con juegos (TIP1), respectivamente.

Figura1- Ejemplos de las categorías de la tarea tradicional T1

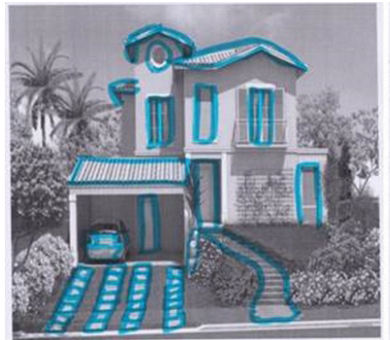

Categoría A

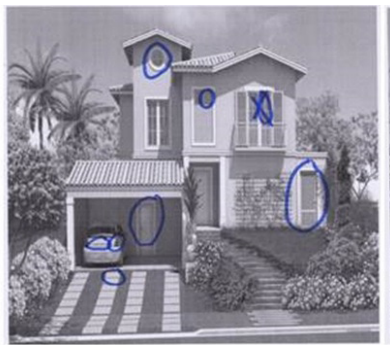

Categoría B

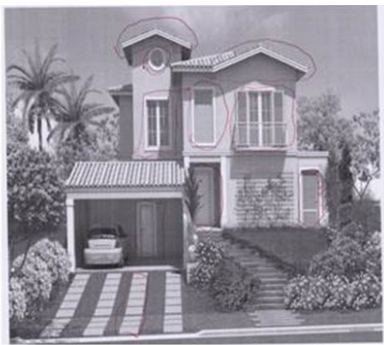

Categoría C

Fuente: Creación propia. 
Figura 2- Ejemplos de las distintas categorías de la tarea con juegos TIP1

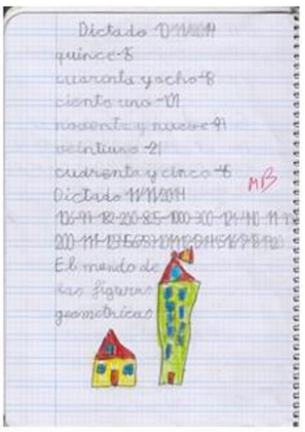

Categoría A

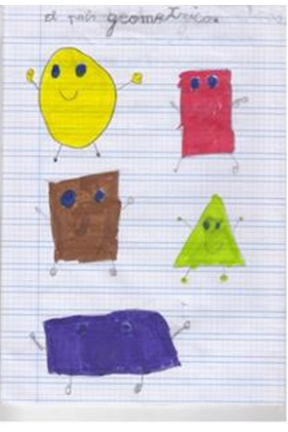

Categoría B

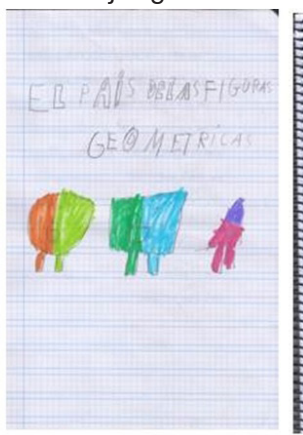

Categoría C

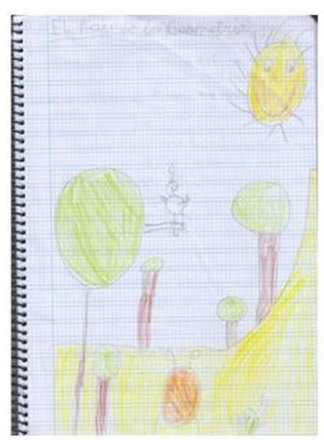

Categoría D

Fuente: Producción original del estudiante en su portafolio.

Tabla 4- Porcentajes en cada categoría para cada tarea de la secuencia didáctica por contenidos y test de Wilcoxon

\begin{tabular}{|c|c|c|c|c|c|c|c|c|c|c|c|c|c|c|}
\hline \multirow[b]{3}{*}{ Contenido } & \multicolumn{5}{|c|}{ Tarea tradicional (\%) } & \multicolumn{5}{|c|}{ Tarea con juegos educativos (\%) } & \multirow{3}{*}{ 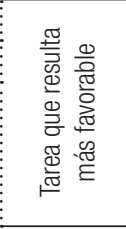 } & \multirow{2}{*}{\multicolumn{3}{|c|}{ Test de Wilcoxon }} \\
\hline & \multirow[b]{2}{*}{$\begin{array}{l}\text { 응 } \\
\text { 잉 }\end{array}$} & \multicolumn{4}{|c|}{ Categorías (\%) } & \multirow[b]{2}{*}{$\begin{array}{l}\text { 음 } \\
\text { 엉 }\end{array}$} & \multicolumn{4}{|c|}{ Categorías (\%) } & & & & \\
\hline & & A & B & C & D & & A & B & C & D & & Z & $\mathrm{p}$ & 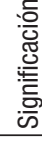 \\
\hline 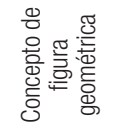 & $\mathrm{T} 1$ & 23,1 & 53,8 & 23,1 & 0 & TIP1 & 15,4 & 46,1 & 7,7 & 30,8 & Tradicional & $-1,508$ & 0,132 & NS \\
\hline 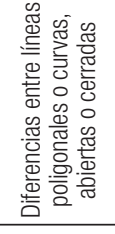 & $\mathrm{T} 2$ & 69,2 & 0 & 15,4 & 15,4 & TIP2 & 53,8 & 38,5 & 7,7 & 0 & Juegos & $-0,724$ & 0,469 & NS \\
\hline 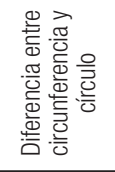 & T3 & 76,9 & 7,7 & 7,7 & 7,7 & TIP3 & 100 & 0 & 0 & 0 & Juegos & $-1,604$ & 0,109 & NS \\
\hline 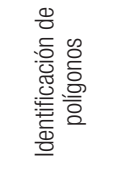 & T4 & 53,8 & 23,1 & 7,7 & 15,4 & TIP4-1 & 76,9 & 23,1 & 0 & 0 & Juegos & $-2,070$ & 0,038 & * \\
\hline & & & & & & TIP4-2 & 100 & 0 & 0 & 0 & Juegos & $-2,201$ & 0,028 & * \\
\hline & T5 & 38,4 & 30,8 & 0 & 30,8 & G5 & 61,5 & 30,8 & 0 & 7,7 & Juegos & $-1,897$ & 0,058 & NS \\
\hline 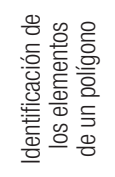 & T6 & 76,9 & 23,1 & 0 & 0 & G6 & 100 & 0 & 0 & 0 & Juegos & $-1,732$ & 0,083 & NS \\
\hline
\end{tabular}

NS: Diferencias estadísticamente no significativas, * Diferencias estadísticamente significativas Fuente: Creación propia. 
Para el concepto de figura geométrica la tarea tradicional (T1) obtuvo mejores resultados de aprendizaje en las tres primeras categorías. Como se deduce de las dos categorías más adecuadas (A y B), el 77\% de los estudiantes fueron capaces de identificar 5 figuras geométricas planas diferentes en la tarea tradicional, y un 61,5\% fue capaz, al menos, de representar todas las que aparecían en el cuento, y en algunos casos $(15,4 \%)$ de combinarlas entre sí.

El ejemplo de la Figura 1 que corresponde a la categoría A para T1, ilustra cómo el alumno marca un triángulo, un cuadrado, un rectángulo, un círculo y un pentágono, algunos de ellos en varios lugares. Por su parte, en la tarea TIP1 se aprecia cómo dibuja una casa donde el tejado es un triángulo, la puerta es un rectángulo, las ventanas son cuadrados y la base principal de la casa está formada por un cuadrado y un triángulo (Figura 2). En la categoría B de la tarea tradicional se puede observar como el alumno identifica más de 5 figuras en la fotografía (Figura 1), aunque varias están repetidas, no siendo capaz de reconocer el pentágono. En la misma categoría de la tarea TIP se aprecia como dibuja un círculo, un cuadrado, un triángulo y dos rectángulos, aunque no es capaz de combinarlos en un mismo dibujo (Figura 2). Lo mismo ocurre en las categorías C y D, donde cada vez el número de figuras identificadas es menor.

A pesar de resultar más favorable la tarea tradicional para este contenido, el test de Wilcoxon no mostró diferencias significativas entre ambas tareas.

\section{Diferencia entre líneas poligonales o curvas, abiertas o cerradas}

Las respuestas de las tareas T2 y TIP2 se analizaron según el número de tipos de líneas (poligonal abierta y cerrada, curva abierta y cerrada) que el estudiante fue capaz de identificar o representar corporalmente: A) 4 tipos, B) 3 tipos, C) menos de 3 tipos, y D) ningún tipo. Las figuras 3 y 4 recogen ejemplos.

Figura 3- Ejemplos de las distintas categorías de la tarea tradicional T2

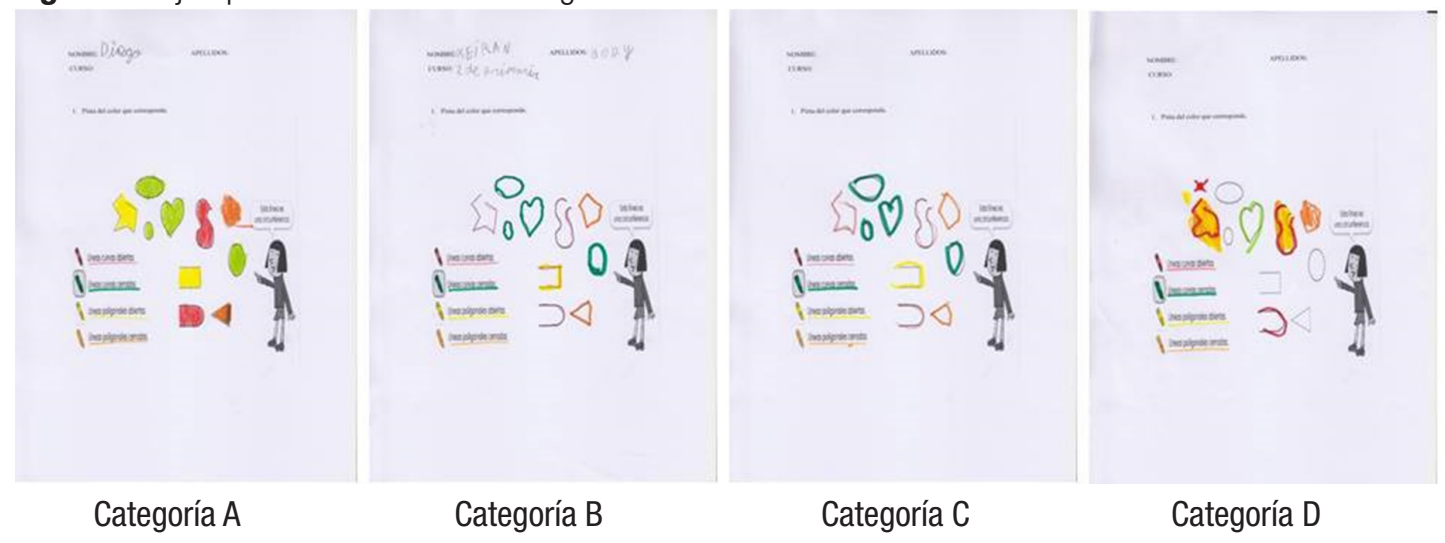

Fuente: Producción original del estudiante en su portafolio. 
Figura 4- Ejemplos de la tarea TIP2
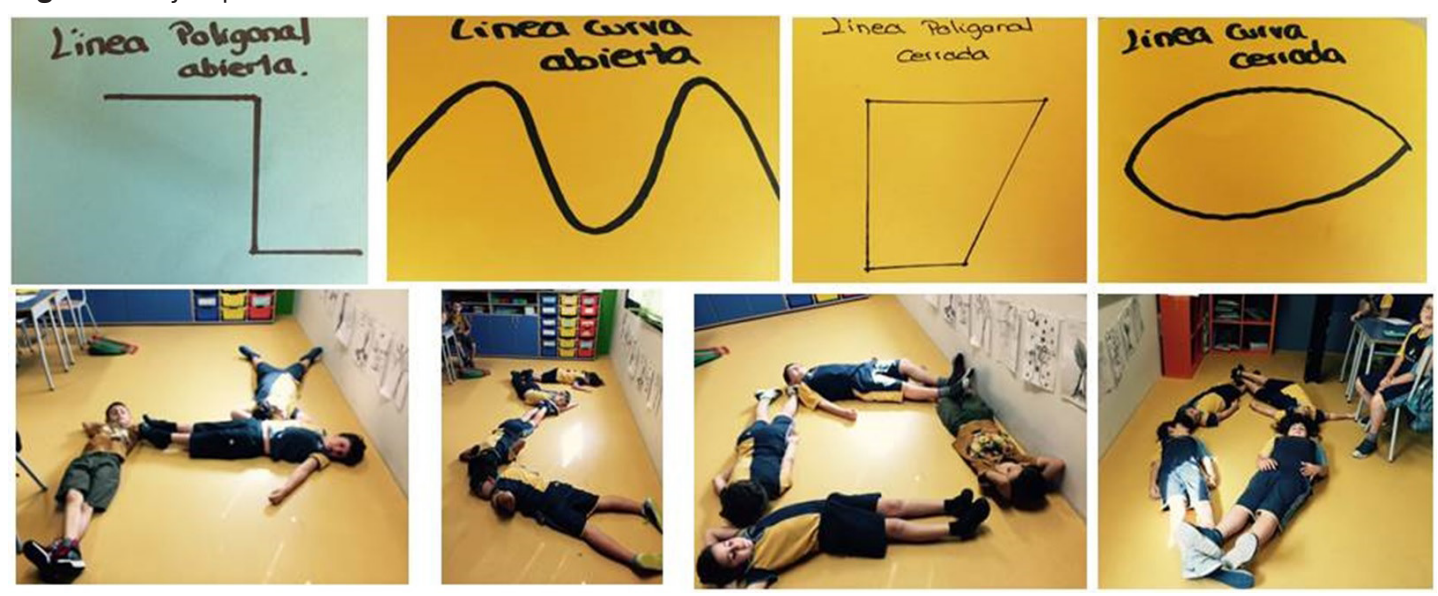

Fuente: Creación propia.

Como se puede observar (Tabla 4), a pesar de que la tarea tradicional presente mejores resultados de aprendizaje en la categoría más adecuada (A), la suma de los porcentajes de las dos primeras categorías de la tarea TIP (92,3\%), en la que se encuentran la mayoría de los estudiantes, hacen que este tipo de recursos sean más favorables. Probablemente esto se deba a que las representaciones de las líneas con el propio cuerpo producen en el alumnado una mayor motivación. Un indicio de ello fueron los comentarios realizados por algunos estudiantes durante su desarrollo, registrados por la profesora en su diario de observación: "Quiero que todas las clases de matemáticas sean así” (Alba) o “¿Cuándo vas a realizar más actividades de este tipo?" (José). Esta hipótesis se refuerza con los resultados de la categoría $\mathrm{D}$, la más inadecuada, donde el porcentaje de alumnos de la tarea tradicional es superior al de la tarea TIP, no habiéndose encontrado ningún caso donde el alumnado fuera incapaz de representar algún tipo de línea. A pesar de detectarse una tendencia favorable hacia esta actividad con juegos, el test de Wilcoxon volvió a mostrar que no existían diferencias significativas entre ambas tareas (Tabla 4).

\section{Diferencia entre circunferencia y círculo}

Las tareas T3 y TIP3 demandaban representar o identificar una circunferencia y un círculo. Sus resultados se categorizaron según la capacidad del estudiante para representar o identificar: A) ambas figuras, B) sólo el círculo, C) sólo la circunferencia o D) ninguna de ellas. La figura 5 presenta algunos ejemplos de las producciones de los alumnos para la tarea tradicional. 
Figura 5- Ejemplos de las distintas categorías de la tarea tradicional T3

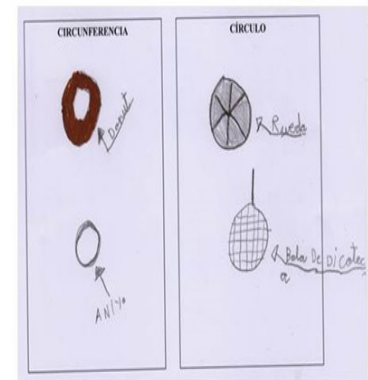

Categoría A

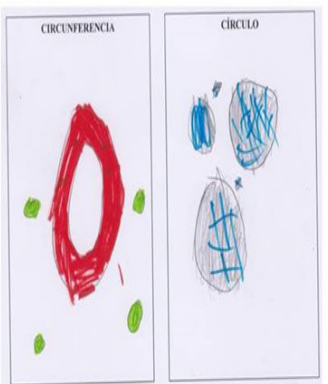

Categoría B

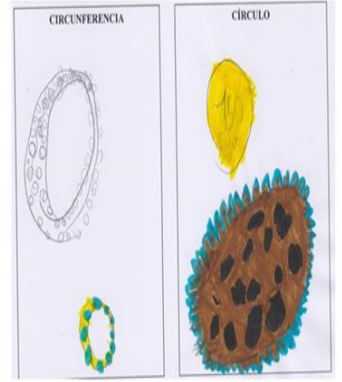

Categoría C

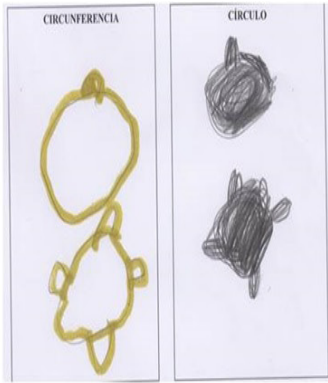

Categoría D

Fuente: Producción original del estudiante en su portafolio.

La mayoría de los estudiantes se sitúan en la categoría A independientemente de que el contenido matemático se presente a través de una tarea TIP o tradicional. No obstante, los mejores resultados se obtienen con TIP, donde la totalidad de respuestas se encuentran en dicha categoría.

Se hace relevante comentar algunos de los dibujos realizados por los estudiantes en la tarea T3. Por ejemplo, se debe tener en cuenta que el alumno reconoce la circunferencia o el círculo desde el plano, es decir, en la superficie del objeto. Así, el alumno clasificado en la categoría A (Figura 5) distingue perfectamente las circunferencias en un donut y un anillo, y el círculo en una rueda o una raqueta de tenis. Sin embargo, el ejemplo de la categoría B ilustra cómo dicho estudiante confunde ambos conceptos al dibujar en la ficha de la circunferencia un flotador y varias pelotas verdes. En el caso de la categoría C se representan como círculos una moneda y el planeta Tierra, pero con una forma ovalada.

Para este contenido, la profesora también registró estos comentarios favorables en su diario sobre la tarea TIP: "ha sido divertido descubrir la forma del objeto sin poder verlo" (Lucía) o "me gusta aprender matemáticas con este método" (Juan). A pesar de ello, el test de Wilcoxon no encontró diferencias estadísticamente significativas (Tabla 4).

\section{Identificación de polígonos}

Para este contenido se propusieron dos bloques de tareas. En el primer bloque, compuesto por tres tareas (T4, TIP4-1 y TIP4-2) el alumno debía identificar o construir un polígono relacionándolo con su nombre, mientras que en el segundo bloque (T5 y G5) trabajaba la división de un polígono en otros.

Las tareas T4, TIP4-1 y TIP4-2 se analizaron en función del número de polígonos que el alumno era capaz de identificar, construir y relacionar con su nombre: A) 5 ó 6 polígonos, B) 3 ó 4 polígonos, C) menos de 3 polígonos, y D) ningún polígono. Las figuras 6, 7 y 8 recogen los resultados de las tareas del primer bloque. 
Figura 6- Ejemplos de las distintas categorías de la tarea tradicional T4

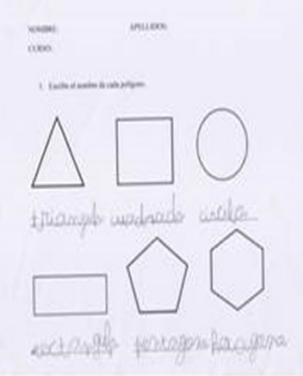

Categoría A

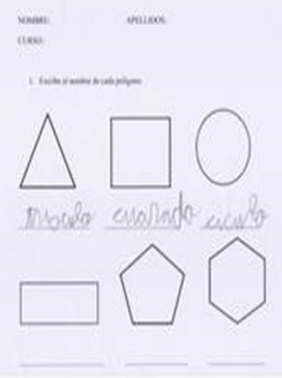

Categoría B

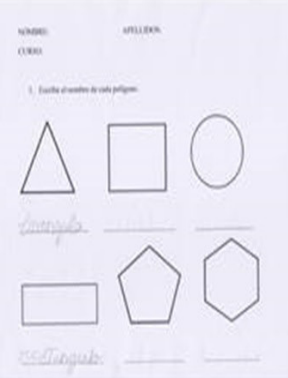

Categoría C

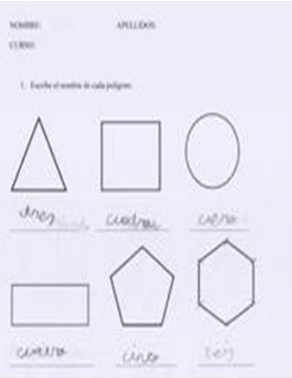

Categoría D

Fuente: Creación propia.

Figura 7- Algunos ejemplos de categorías de la tarea TIP4-1

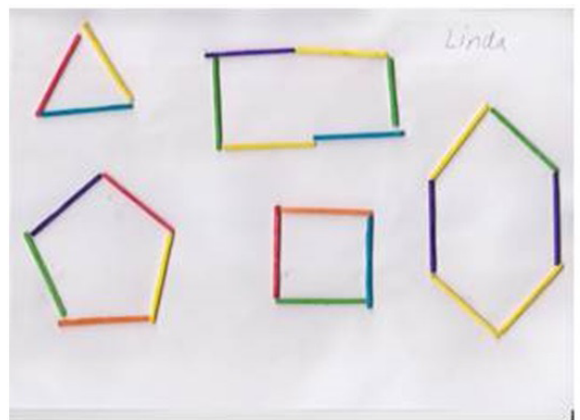

Categoría A

Fuente: Creación propia.

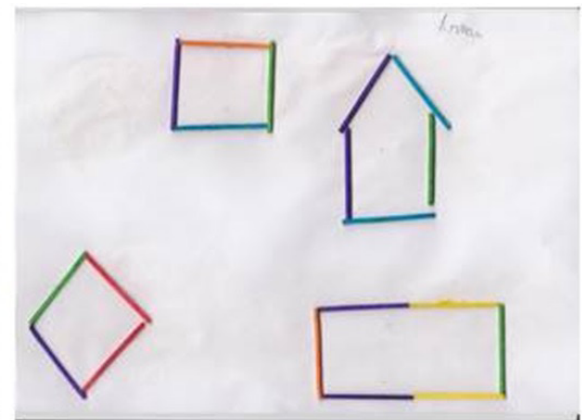

Categoría B

Figura 8- Algunos ejemplos de la tarea TIP4-2
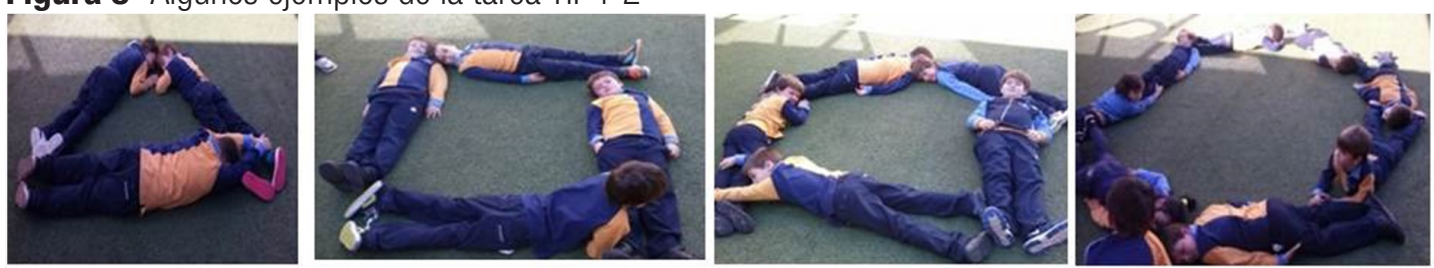

Fuente: Creación propia.

De nuevo, los mejores resultados se encuentran en las dos tareas TIP (Tabla 4) en las que todos los estudiantes se sitúan en las dos primeras categorías (A y B), a diferencia de la tarea tradicional donde se distribuyen entre las cuatro categorías. Probablemente el uso de escenarios motivadores como palillos de colores o la expresión corporal para construir polígonos contribuyan de forma favorable a la adquisición de este aprendizaje. Los comentarios de varios alumnos así lo resaltaban: "En esta clase hacemos deporte y matemáticas a la vez" (Ana). Del mismo modo, la siguiente reflexión de la profesora lo 
evidenciaba: "El uso de estos escenarios hacen que los alumnos realicen la tarea con agrado, lo que favorecerá sin duda su aprendizaje” (Diario del profesor).

El segundo bloque de este contenido (T5 y G5) solicitaba dividir o representar una figura dada formada por otras. Sus resultados se ajustaron a las siguientes categorías según la capacidad del estudiante de: A) dividir o construir una/varias figura/s dada/s a partir de otras, B) resolver una parte importante de la tarea adecuadamente, C) presentar más errores que aciertos en su resolución, y D) no fuera capaz de dividir o construir ninguna parte de una figura dada. Las figuras 9 y 10 ilustran las producciones de los estudiantes.

Figura 9- Ejemplos de las distintas categorías de la tarea tradicional T5

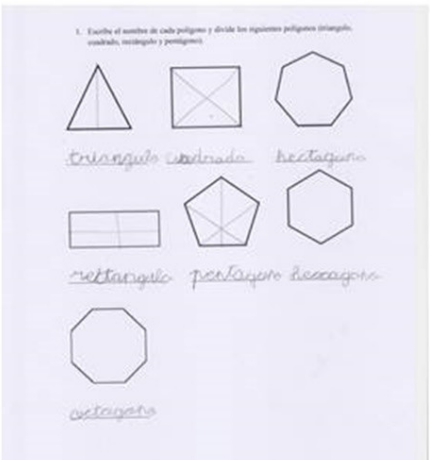

Categoría A

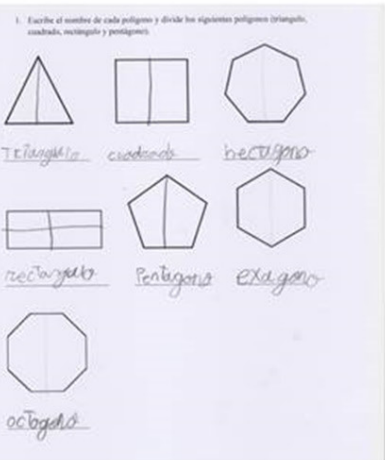

Categoría B

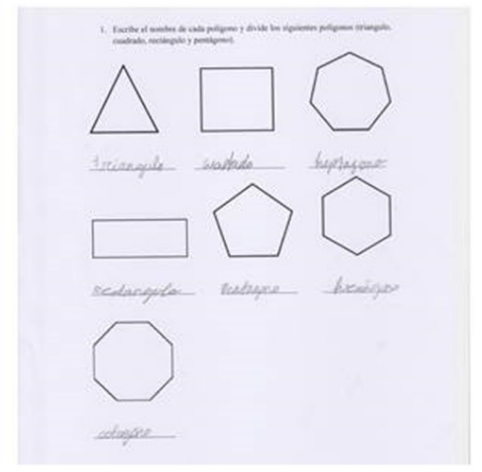

Categoría D

Fuente: Producción original del estudiante en su portafolio.

Figura 10- Ejemplos de algunas de las categorías de la tarea G5

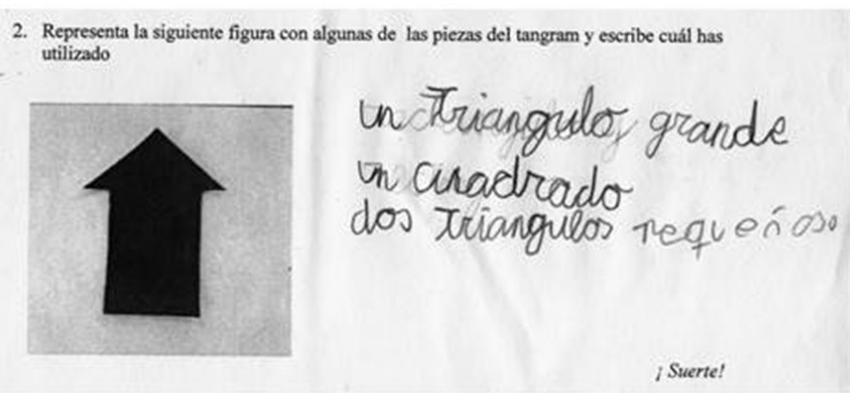

Categoría A

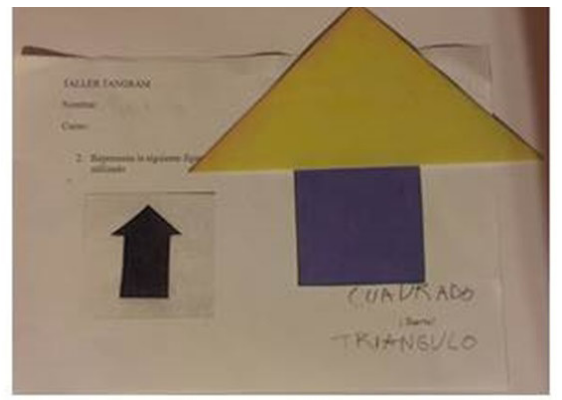

Categoría B

Fuente: Producción original del estudiante en su portafolio.

Para estas tareas, un gran porcentaje de las respuestas se concentró en las categorías A y B, siendo mayoritarios los porcentajes obtenidos en la tarea G5 del tangram (92,3\% de los estudiantes) (Tabla 4). En la tarea tradicional se puede observar cómo el alumno divide adecuadamente en las partes que se piden los polígonos más sencillos (Figura 9) y es capaz de ofrecer diferentes posibilidades. Así, en la categoría A se observa cómo un 
alumno ha dividido el cuadrado en cuatro triángulos y en la categoría $\mathrm{B}$, otro estudiante divide la misma figura en dos rectángulos. Sin embargo, la mayoría del alumnado no es capaz de dividir con éxito polígonos con más de cinco lados. Por el contrario, parece resultar más sencilla la combinación de algunos polígonos para formar la figura con las piezas del tangram. Así, 8 alumnos son capaces de combinar cuatro polígonos (triángulo grande, dos triángulos pequeños y un cuadrado) para formar la figura propuesta. Como se observa, en el ejemplo de la categoría B (Figura 10), otros estudiantes intentan construir una forma parecida con un triángulo y un cuadrado.

A pesar de detectarse comentarios favorables hacia las tareas con juegos educativos como "las actividades con juegos me encantan" (Darío), al avanzar las sesiones fueron también frecuentes comentarios desfavorables hacia el uso de tareas tradicionales: “¿Por qué tenemos que seguir haciendo fichas?” (Luís), “Jo... estas actividades [las tradicionales] no me gustan, prefiero las otras [se refiere a las tareas con juegos]” (Alejandro) o “¿cuándo se termina la clase?" (Iván), comentarios indicativos de que este tipo de tareas les resultaban más aburridas y menos motivadoras.

Como se aprecia en la Tabla 4, sólo para este contenido el test de Wilcoxon mostró diferencias significativas al comparar las parejas de tareas T4 y TIP4-1, por un lado, y T4 y TIP4-2, por otro. Sin embargo, la comparación T5 y G5 mostró solo una cierta tendencia a la significatividad $(\mathrm{Z}=-1,897 ; \mathrm{p}=0,058)$. Esto pone de manifiesto, al menos, con la muestra estudiada, que TIPs parecen resultar más favorables que games para aprender este contenido.

\section{Identificación de los elementos de un polígono}

Las tareas T6 y G6 se analizaron según el número de figuras geométricas en las que era capaz de identificar sus elementos: A) 5 figuras, B) 3 ó 4 figuras, C) menos de 3 figuras, y D) ninguna figura. La figura 11 muestra resultados de la tarea T6, mientras que la figura 12 recoge los materiales del juego educativo.

Figura 11- Ejemplos de la tarea tradicional T6

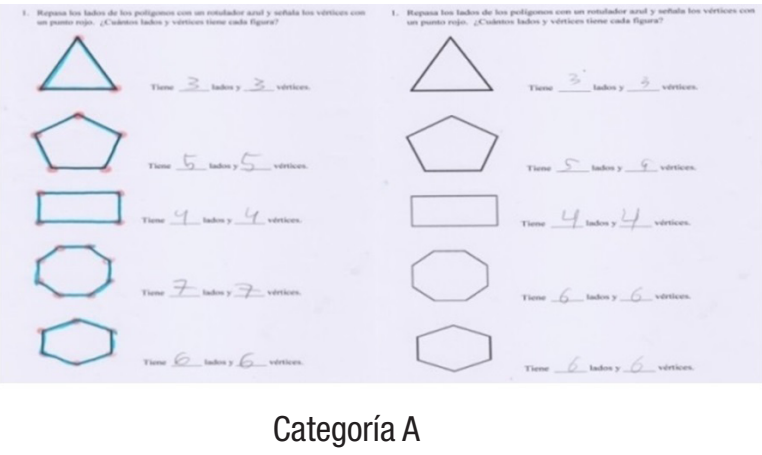

Fuente Creación propia.
Figura 12- Materiales de la tarea G6

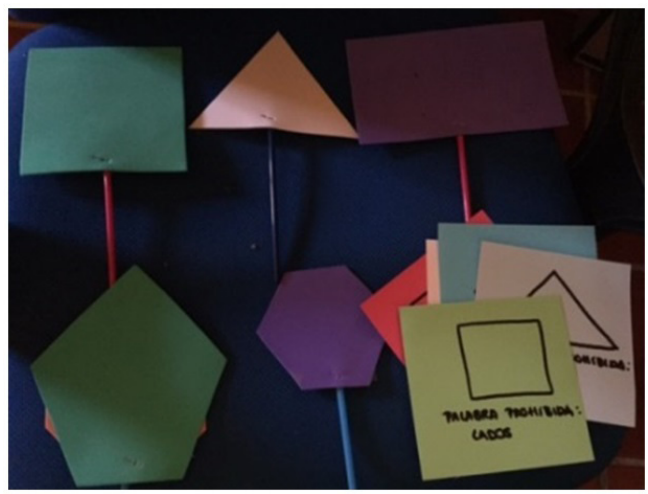

Categoría B

Fuente Creación propia. 
Los resultados muestran que gran parte del alumnado resuelve la tarea de forma adecuada (categoría A), independientemente que se haya planteado de forma tradicional o a través de juegos, como confirma que casi el 77\% y el 100\% de alumnos se sitúen en la categoría A en T6 y G6 respectivamente, y que no se encuentren resultados en las categorías $\mathrm{C}$ y D. Como en ocasiones anteriores, el juego parece resultar más exitoso que la tarea tradicional. A pesar de ello, no se encontraron diferencias significativas en el test de Wilcoxon para este contenido.

Finalmente, otros indicios de los beneficios del uso de juegos educativos en el aula se encuentran en el diario de la profesora, a través de estas reflexiones:

En el desarrollo de las tareas con juegos he observado un incremento de la atención de los estudiantes y de la confianza en sí mismos al dominar los nuevos conocimientos, habilidades o estrategias. A diferencia de las actividades tradicionales, las tareas lúdicas rompieron con la monotonía, aumentaron la motivación, mejoraron las relaciones sociales entre ellos y crearon un clima de clase muy apropiado, incluso también fuera del aula donde se desarrollaron algunas de estas tareas (Diario de la profesora observadora).

El grado de participación del alumnado a lo largo de la unidad, se manifiesto en que los estudiantes finalizaron las tareas propuestas, independientemente de su naturaleza, aunque las actividades TIPs y con games se realizaron en menor tiempo.

\section{Conclusiones}

Los resultados obtenidos en este estudio preliminar parecen indicar que el uso de un enfoque basado en juegos educativos integrados en una secuencia didáctica sobre geometría puede contribuir de forma favorable al aprendizaje de alumnos de 7 años en comparación con una metodología más tradicional con fichas de trabajo y escasas oportunidades en el contexto social (pregunta de investigación 1), al haberse encontrado diferencias estadísticamente significativas en el contenido de identificación de polígonos.

Asimismo, los resultados parecen apuntar que el tipo de juego educativo que parece ser más efectivo en el aprendizaje de la geometría (TIP o game) (pregunta de investigación 2), son los TIPs ya que sólo en éstos se obtuvieron diferencias estadísticamente significativas al compararlos con tareas tradicionales.

Estos resultados deben considerarse con cautela, al tratarse de una secuencia puntual con una muestra de estudiantes muy reducida en un estudio preliminar, que se hacía necesaria para profundizar en el seguimiento de las tareas de los alumnos como estudio de caso, y que ha constituido también la principal limitación del estudio. Como muestran otros estudios, el aprendizaje no se demuestra necesariamente en el momento en que se realiza la actividad y puede ser en momentos posteriores en otros contextos donde una determinada noción se llega a movilizar.

En este sentido, los resultados obtenidos y la apreciación global a nivel de porcentajes demuestran que las tareas con juegos educativos parecen producir avances en el aprendizaje respecto a tareas tradicionales en la mayoría de los contenidos de geometría estudiados. Estos resultados nos animan a realizar estudios posteriores con muestras más amplias potenciando el uso de recursos TIPs en la enseñanza de las matemáticas, ya que probablemente se obtengan diferencias significativas en este sentido. Finalmente, el uso 
de games en dos contenidos (identificación de polígonos y elementos de polígonos) que mostraron una tendencia estadística muy próxima a la significatividad, nos hace pensar que también este tipo de recursos pueda favorecer el aprendizaje de las matemáticas si son cuidadosamente diseñados.

En definitiva, los resultados parecen determinar que un enfoque basado en juegos educativos en el aula puede constituir una estrategia de interés para fomentar el aprendizaje y la motivación, por lo que los docentes deben promover metodologías innovadoras que incluyan, entre otros recursos, algunas de este tipo. De esta forma, se facilitará al estudiante un acercamiento de las matemáticas a la vida cotidiana, desarrollando competencias, estrategias y habilidades prácticas.

\section{Referencias}

ALSINA, Claudi. Educación matemática e imaginación. Unión, Revista Iberoamericana de Educación Matemática, Santiago de Chile, v. 11, p. 9-17, 2007.

ALSINA, Claudi; FORTUNY, Josep María; PÉREZ, Rafael. ¿Por qué geometría? Propuestas didácticas para la E.S.O. Madrid: Síntesis, 1997.

BAKHTIN, Mikhail. Rabelais and his world. Bloomington: Indiana University Press, 1984.

BELANICH, James; SIBLEY, Daragh; ORVIS, Kara. Instructional characteristics and motivational features of a PC-based game. Alexandria: Army Research Institute for the Behavioral and Social Science, 2004. (ARI research report 1822).

BLANCO, Ángel. Relaciones entre la educación científica y la divulgación de la ciencia. Revista Eureka sobre Enseñanza y Divulgación de las Ciencias, Cádiz, v. 1, n. 2, p. 70-86, 2004.

BLANCO, Ángel et al. Key aspects of scientific competence for citizenship: a delphi study of the expert community in Spain. Journal of Research in Science Teaching, Netherlands, v. 52, n. 2, p. 164-198, 2015.

BRUNER, Jerome. Juego, pensamiento y lenguaje. Perspectivas, Ginebra, v. 16, n. 1, p. 79-85, 1986.

BUENO, Ana; MONTEOLIVA, Miguel. Fotografía y matemáticas: una experiencia en la Axarquía. Epsilon, Cádiz, v. 27, p. 63-68, 1993.

CAILLOIS, Roger. Man, play, and games. Chicago: University of Illinois Press, 2001.

CANO, Natalia Andrea; ZAPATA, Fabio Nelson. La enseñanza de las matemáticas a través de la implementación del juego del rol y de aventura. Unión, Revista Iberoamericana de Educación Matemática, Santiago de Chile, v. 23, p. 211-222, 2010.

CHAMOSO, José et al. Análisis y experimentación de juegos como instrumentos para enseñar matemáticas. Suma, Badalona, v. 47, p. 47-58, 2004.

CHAZAN, Saralea. Profiles of play: assessing and observing structure and process in play therapy. London: Jessica Kingsley, 2002. 
CLEMENTS, Douglas. Playing math with young children. Curriculum Administrator, Trumbull, v. 35, n. 4, p. 25-28, 1999.

CONFREY, Jere. What constructivism implies for teaching'. Journal for Research in Mathematics Education, Phoenix, v. 4, p. 107-122, 1992.

CORBALÁN, Fernando. Juegos matemáticos para secundaria y bachillerato. Madrid: Síntesis, 1994.

CORBALÁN, Fernando; DEULOFEU, Jordi. Juegos manipulativos en la enseñanza de las matemáticas. Uno, Revista de Didáctica de las Matemáticas, Barcelona, v. 7, p. 71-80, 1996.

DE GUZMÁN, Miguel. Juegos matemáticos en la enseñanza. Suma, Badalona, v. 59, p. 5-38, 2004.

DEMPSEY, John V. et al. Forty simple computer games and what they could mean to educators. Simulation and Gamming, New York, v. 33, n. 2, p. 157-168, 2002.

DETERDING, Sebastian et al. From game design elements to gamefulness: defining "gamification". In: INTERNATIONAL ACADEMIC MINDTREK CONFERENCE, ENVISIONING FUTURE MEDIA ENVIRONMENTS, 15., 2011, New York. Proceedings... New York: ACM, 2011. p. 9-15

DRIVER, Rosalind; OLDHAM, Valerie. Constructivist approach to curriculum development in science. Studies in Science Education, London, v. 13, p. 105-122, 1986.

DURÁN, Jesús et al. Análisis y experimentación de juegos como instrumentos para enseñar matemáticas. Suma, Badalona, v. 47, p. 47-58, 2004.

EBNER, Martin; HOLZINGER, Andreas. Successful implementation of usercentered game based learning in higher education: An example from civil engineering. Computer and Education, Amsterdam, v. 49, n. 3, p. 873-890, 2007.

ERNEST, Paul. The dialogical nature of mathematics. In: ERNEST, Paul (Ed.). Mathematics, education and philosophy: an international perspective. 1.ed. London: Falmer Press, 1994. p. 33-48.

ERNERST, Paul. The nature of mathematics and teaching. Philosophy of Mathematics Education Journal, Exeter, v. 9, p. 46-53, 1996.

ERNEST, Paul. The philosophy of mathematics education. London: Falmer Press, 1991.

ERNEST, Paul. What is the philosophy of mathematics education? Philosophy of Mathematics and Education Journal, Exeter, v. 18, p. 1-16, 2004.

ESPAÑA. Ministerio de Educación, Cultura y Deporte. Real Decreto 126/2014, de 28 de febrero, por el que se establece el currículo básico de la educación primaria. BOE, Madrid, n. 52, p. 19349, mar. 2014.

FARIAS, Deninse; ROJAS, Freddy. Estrategias lúdicas para la enseñanza de la matemática en estudiantes que inician estudios superiores. Revista Paradigma, Maracay, v. 31, n. 2, p. 53-64, 2010.

FRANCO-MARISCAL, Antonio Joaquín; VIDAL, Fernando. Fútbol y combinatoria: algunas ideas para trabajar la competencia matemática en secundaria. Uno, Revista de Didáctica de las Matemáticas, Barcelona, v. 62, p. 94-101, 2013. 
Un enfoque basado en juegos educativos para aprender geometría en educación primaria. Estudio...

FRANCO-MARISCAL, Antonio Joaquín et al; A game-based approach to learning the idea of chemical elements and their periodic classification. Journal of Chemical Education, Washington, D.C., v. 93, p. 1173-1190, 2016.

GOODALE, Thomas L.; GODBEY, Geoffrey. The evolution of leisure: historical and philosophical perspectives. State College: Venture, 1988.

HANDAL, Boris. Philosophies and pedagogies of mathematics. Philosophy of Mathematics Education Journal, Exeter, v. 17, 2003.

HENRICKS, Thomas. Play as ascending meaning: implications of a general model of play. In: REIFEL, Stuart (Ed.). Play contexts revisited. Stamford: Ablex, 1999. p. 257-277.

HUIZINGA, James. Homo ludens: a study of play-element in culture. London: Routledge, 2000.

INTERNATIONAL CONGRESS MATHEMATICS I. Perspectives on the teaching of geometry for the 21st century. Discussion Document for an ICMI study. In: C. MAMMANA, Carmelo; VILLANI, Vinicio (Ed.). Perspectives on the teaching of geometry for the 21st century: an ICMI study. Dordrecht: Kluwer, 1998. p. 337-345.

MILLAR, Susanna. Psicología del juego infantil. Barcelona: Fontanella, 1992. (Conducta humana; n. 9).

MINERVA TORRES, Mónica. El juego: una estrategia importante. Educere, Revista Venezolana de Educación, Mérida, v. 6, n. 19, p. 289-296, 2002.

MINERVA TORRES, Mónica. El juego en el aula: una experiencia de perfeccionamiento docente en matemática a nivel institucional. Suma, Badalona, v. 38, p. 23-29, 2001.

MONDEJA, Diana et al. Juegos didácticos: ¿útiles en la educación superior?. Pedagogía Universitaria, La Habana, v. 6, n. 3, p. 65-76, 2001.

MUÑIZ, Laura; ALONSO, Pedro; RODRÍGUEZ, Luís. El uso de los juegos como recursos didácticos para la enseñanza y el aprendizaje de las matemáticas: estudio de una experiencia innovadora. Unión, Revista Iberoamericana de Educación Matemática, Santiago de Chile, v. 39, p. 19-33, 2014.

NACIONES UNIDAS. Asamblea general. Programa de acción mundial para los jóvenes hasta el año 2000 y años subsiguientes. Resolución aprobada por la Asamblea General [sin remisión previa a una Comisión Principal (A/50/728)] 50/81, 1996. Disponible en: <http://www.cinu.mx/minisitio/UNjuventud/docs/ Programa_accion_mundial_jovenes.pdf>.Acceso: 06 feb.2018.

OHIO DEPARTMENT OF EDUCATION. Ohio's early learning and development standards: birth to kindergarten entry, 2004. Disponible en: <http://education.ohio.gov/getattachment/Topics/Early-Learning/EarlyLearning-Content-Standards/Birth-Through-Pre_K-Learning-and-Development-Stand/ELSD-CognitionStandards.pdf.aspx>. Acceso: 06 feb.2018.

OLFOS, Raimundo; VILLAGRÁN, Eduvina. Actividades lúdicas y juegos en la iniciación al álgebra. Íntegra, Diego Portales, v. 5, p. 1-13, 2001.

ORGANIZATION FOR ECONOMIC CO-OPERATION AND DEVELOPMENT. PISA 2015 Assessment and analytical framework: science, reading, mathematic and financial literacy. Paris: OECD, 2016.

ORLIK, Yuri. Chemistry: active methods of teaching and learning. México, DF: Iberoamérica, 2002. 
OSBORNE, Jonathan. Teaching critical thinking?. New directions in science education. School Science Review, Herts, v. 352, p. 53-62, 2014.

ROJAS, Iván Roberth. Aplicación de juegos lógicos en Juventud Salesiana. Unión, Revista Iberoamericana de Educación Matemática, Santiago de Chile v. 19, p. 150-156, 2009.

SALCEDO, Betty. Cuento "El país de las formas geométricas". En Proyecto pedagógico de aula Aprendamos las figuras geométricas. Escuela rural mixta San Agustín, 2012. Disponible en: <https://www.youtube.com/ watch?v=U9KHX7wfw7E >. Acceso: 06 feb.2018.

SALEN, Katie; ZIMMERMAN, Eric. Rules of play: game design fundamentals. Cambridge: MIT, 2004.

VARELA, Laura; GRADAÍLLE, Rita; TEIJEIRO, Yésica. Ocio y usos del tiempo libre en adolescentes de 12 a 16 años en España. Educação e Pesquisa, São Paulo, v. 42, n. 4, p. 987-999, out./dez. 2016.

VILLARROEL, Silvia; SGRECCIA, Natalia. Enseñanza de la geometría en secundaria. Caracterización de materiales didácticos concretos y habilidades geométricas. Unión, Revista Iberoamericana de Educación Matemática, Santiago de Chile v. 29, p. 59-84, 2011.

WAYER, Nicola; CRIPPEN, Kent; DAWSON, Kara. Design and enactment of online components during four blended learning courses. Journal of Online Learning Research, Waynesville v. 1, n. 2, p. 219-239, 2015.

Recibido en: 16.08 .2017

Revisiones en: 06.02.2018

Aprobado en: 15.05 .2018

Antonio-Joaquín Franco-Mariscal: Licenciado y Doctor en Química por la Universidad de Cádiz, profesor de la Facultad de Ciencias de la Educación, Didáctica de las Ciencias Experimentales de la Universidad de Málaga (España) y profesor de enseñanza secundaria en excedencia. Sus líneas de investigación son gamificación, competencia científica, matemática y prácticas científicas.

Paola Simeoli Sánchez: Técnica Superior de Formación Profesional en Educación Infantil graduada en Educación Primaria con mención en Escuela inclusiva y atención a la diversidad por la Facultad de Ciencias de la Educación, Didáctica de las Ciencias Experimentales de la Universidad de Málaga (España). Actualmente está desarrollando su labor profesional en el campo de las discapacidades cognitivas en el Reino Unido. 